\title{
Density expansion for the mobility in a quantum Lorentz model
}

\author{
K. I. Wysokiński \\ Institute of Physics, \\ M.Curie-Sktodowska University, \\ 20-031 Lublin, Poland \\ and \\ Materials Science Institute \\ University of Oregon \\ Eugene, OR 97403 \\ Wansoo Park and D. Belitz \\ Department of Physics and Materials Science Institute, \\ University of Oregon, \\ Eugene, OR 97403 \\ T. R. Kirkpatrick \\ Institute for Physical Science and Technology, \\ University of Maryland, \\ College Park, MD 20742
}

(April 1, 2018)

\begin{abstract}
We consider the mobility of electrons in an environment of static hard-sphere scatterers, which provides a realistic description of electrons in Helium gas. A systematic expansion in the scatterer density is carried to second order relative to the Boltzmann result, and the analytic contribution at this order is derived, together with the known logarithmic term in the density expansion. It is shown that existing experimental data are consistent with the existence of the logarithmic term in the density expansion, but more precise experiments are needed in order to unambiguously detect it. We show that our calculations provide the necessary theoretical information for such an experiment, and give a detailed discussion of a suitable parameter range.
\end{abstract}

PACS numbers: 51.10+y, 05.60+w 


\section{INTRODUCTION}

It has been known for almost thirty years that transport coefficients, in contrast to thermodynamic quantities, do not possess a virial expansion. 1 This insight came originally

as a substantial surprise, and the physics behind it turned out to be very fundamental in nature. Namely, it is related to the, previously unexpected, existence of long-range dynamical correlations in equilibrium fluids. As a specific example, let us consider the diffusion of a tagged particle in a fluid. The classic method for setting up a virial or density expansion for the diffusivity was based on a generalized Boltzmann equation. In this method one constructs a virial expansion for the collision operator by taking into account collisions of the tagged particle with a successively increasing number of scatterers which is, however, finite at every order in the expansion. This is analogous to the Ursell-Mayer cluster expansion for thermodynamic guantities.4 Alternatively, one can use the Green-Kubo or time correlation function method. 3 Both methods yield identical formal results for the density expansion of the diffusivity $D$ (or any other transport coefficient),

$$
D / D_{B}=1+D_{1} n+D_{2} n^{2}+O\left(n^{3}\right),
$$

where $D_{B}$ is the Boltzmann result for the diffusivity, $n$ is the dimensionless density of the fluid, and $D_{1}, D_{2}$, etc. are the 'virial coefficients'. The surprise consisted in the realization that the coefficients in this expansion are infinite past a certain order that depends on the dimensionality of the system.6 It was soon realized that these infinities signalize the presence of a logarithmic density dependence in the expansion given by Eq. (1.1). F For instance, in a three-dimensional (3-d) system, the coefficient $D_{2}$ is $n$-dependent, and goes for small $n$ like $D_{2} \sim \ln n+$ const, so that Eq. (1.1) must be replaced by,

$$
D / D_{B}=1+D_{1} n+D_{2 l o g} n^{2} \ln n+D_{2} n^{2}+o\left(n^{2}\right)
$$

where $D_{2 l o g}$ and $D_{2}$ are numbers, and $o\left(n^{2}\right)$ denotes terms that vanish faster than $n^{2}$ for $n \rightarrow 0$. The functional form of the higher coefficients is not known. The physical reason for the breakdown of the density expansion, and the appearance of the logarithmic terms, is a collective effect: Ring collisions, in which the scattered particle collides with a scatterer, and then visits a number of other scatterers before returning to the original one, turn out to lead to infinite phase space volumes if a finite number of scatterers is considered, and hence the distances between scatterers, and the times traveled between them, are allowed to become arbitrarily large. These collision events are, however, unphysical, because the inevitable presence of scatterers that do not belong to the particular cluster under consideration does not allow the effective distance traveled between successive scattering events to be substantially longer than a mean-free path. Properly taking into account this meanfree-path damping of the scattered particle's trajectory leads to the mentioned logarithmic term. The latter represents dynamic correlations in the fluid which extend over a distance of a few mean-free paths (or a time equal to a few mean-free times), which in the limit of small densities becomes much larger than the effective particle size, or the range of the intermolecular force. In contrast, static correlations in classical equilibrium fluids extend only over the range of the intermolecular force, which is the reason why the cluster expansion works for thermodynamic quantities. 
Due to its bearing on fundamental aspects of the Statistical Mechanics of both equilibrium and non-equilibrium systems, and its close connection to related effects, such as long-time tails, 1 the breakdown of the density expansion for transport coefficients has generated substantial interest over almost thirty years. The existence of the logarithmic term has been ascertained theoretically for a variety of classical systems, both real fluids and model fluids, and for a variety of transport coefficients. simulations of a 2-d Lorentz gas, 8 where the logarithm appears at first order rather than at second order due to the lower dimensionality. It has also been established that the same effect occurs for quantum mechanical scattered particles. 9 However, its experimental verification has proven to be extraordinarily difficult.10 The reasons for this are manifold. First, the detection of any logarithmic term on an analytic background is very difficult due to the slow variation of the logarithm. Second, for 3-d systems the effect appears only at second order in the density expansion beyond the Boltzmann equation. Third, the coefficients in Eq. (1.2), or in the equivalent expressions for other transport coefficients, are not known for classical fluids with realistic interaction potentials. Even for a hard-core model fluid it has so far not been possible to calculate the coefficient $D_{2}$ of the analytic term at second order. Since the detection of a logarithmic term on an unknown background is a hopeless task, this essentially precludes the use of 3-d classical fluids for a convincing observation. On the other hand, 2-d classical systems are very hard to realize. Furthermore, estimates of the values of the coefficients in classical systems show that for those transport coefficients that can be accurately measured, like e.g. the shear viscosity, the coefficient of the analytic second order term is much larger than the one of the logarithmic term. This is because the former contains essentially excluded volume effects, which dominate over the ring collision contributions.

These difficulties raise the question whether quantum systems are possibly better suited for an experimental verification of the logarithmic term than classical ones. 2-d quantum systems, although easy to realize, are not suitable since transport in 2-d quantum systems is pathological due to localization effects.11.12 This leaves 3-d quantum systems. Here the effect also occurs only at second order, but otherwise the situation is much more favorable than in the case of 3-d classical systems, as we will see. A chief advantage is the fact that the hard-core Lorentz gas model, i.e. a tagged particle moving in an array of static hardsphere scatterers, 13 is a much better approximation for certain quantum systems than for any classical ones. 3-d quantum systems are therefore the most promising candidates for an experimental observation of the nonanalyticity.

A particularly promising system consists of electrons injected into Helium gas of density $n .14$ The electron-Helium scattering process is well known, and its characteristics are convenient from a theoretical point of view. The scattering length, $a_{s}=0.63 \AA$, is positive, and for thermal electrons the energy dependence of the scattering cross section is negligible. Since the electrons behave quantum mechanically, the thermal wavelength, $\lambda=\left(2 \pi^{2} \hbar^{2} \beta / m\right)^{1 / 2}$, with $\beta=1 / k_{B} T$ and $m$ the electron mass, provides an additional length scale besides $a_{s}$ and the mean Helium atom separation $n^{-1 / 3}$. The leading parameter in the density expansion is $n a_{s}^{2} \lambda=\lambda / 4 \pi l, 15$ with $l=1 / 4 \pi n a_{s}^{2}$ the mean free path, and $a_{s} / \lambda$ serves as an additional small parameter. The mass ratio $m_{H e} / m \approx 10^{4}$ makes it a good approximation to treat the Helium atoms as static scatterers. Finally, the low density of the injected electrons allows one to neglect Coulomb interaction effects between the electrons. An experiment 14 which 
measures the mobility of the electrons (which is related to the diffusion coefficient by an Einstein relation) thus constitutes an almost ideal realization of a $3-d$ quantum Lorentz model.

The density expansion for the transport coefficients of the $3-d$ quantum Lorentz model has been considered in Refs. 15, 16. The experimentally relevant quantity is the mobility, $\mu$, at finite temperature. The leading terms in the expansion for $\mu$, analogous to Eq. (1.2), are,

$$
\mu / \mu_{B}=1+\mu_{1} \chi+\mu_{2 \log } \chi^{2} \ln \chi+\mu_{2} \chi^{2}+O\left(\chi a_{s} / \lambda\right)+o\left(\chi^{2}\right),
$$

with

$$
\begin{gathered}
\mu_{1}=-\pi^{3 / 2} / 6, \\
\mu_{2 l o g}=\left(\pi^{2}-4\right) / 32 .
\end{gathered}
$$

Here $\mu_{B}(T)=(4 e l / 3)(2 \pi m / \beta)^{-1 / 2}$ is the Boltzmann mobility, and $\chi=\lambda / \pi l$ and $a_{s} / \lambda$ are small parameters.

Adams et al.17 have used Eqs. (1.3) to analyze experimental data obtained from timeof-flight measurements for electrons in $\mathrm{He}$ and $\mathrm{H}_{2}$. Their main objective was to refute the popular misconception that $\mu_{1}=\mu_{2 l o g}=0,18$ which arose from an inappropriate application of localization ideas to the low-density regime. Ref. 17 concluded that the existing experiments give very good agreement with the value of $\mu_{1}$ given in Eq.(1.3b). This success raised the question whether the same system could be used to observe the logarithmic term. In the absence of information about $\mu_{2}$ this would involve measuring the conductivity over a gas density range that is sufficient to observe the logarithmic dependence directly. This is clearly hopeless. However, if $\mu_{2}$ was known, then the logarithmic term would just provide a weakly density dependent correction to it, and a sufficiently accurate experiment at fixed gas density would be sufficient to probe the existence of the logarithmic term. This background provided the motivation for a calculation of $\mu_{2}$, the result of which has been reported in a recent short communication as, 19

$$
\mu_{2}=0.236 \ldots
$$

The purpose of the present paper is to provide the technical details of the calculation whose result was reported in Ref. 19. We will also give a more detailed discussion of how our result can be used to design an experiment capable of observing the logarithmic term. The paper is organised as follows. In Section [1] we first define the model, and set up a diagrammatic perturbation theory for the conductivity at zero temperature. We then perform the calculation to second order in the scatterer density, and convert the result into an expression for the experimentally relevant temperature dependent mobility. Section 【II contains a detailed discussion of the relevance of our results for a proposed experiment to detect the logarithmic term in the density expansion. Some technical details are relegated to two appendices. 


\section{DENSITY EXPANSION FOR THE MOBILITY}

\section{A. The Model}

We consider a model of noninteracting electrons moving in three dimensional space, and scattering off static, uncorrelated, and randomly located impurities with density $n$. The electron-impurity interaction can be characterised by the scattering cross section $\sigma_{s}=4 \pi a_{s}^{2}$, where $a_{s}$ is the scattering length.

The Hamiltonian for this system is,

$$
H=\sum_{\mathbf{k}, \sigma}\left(\epsilon_{\mathbf{k}}-\mu\right) a_{\mathbf{k}, \sigma}^{\dagger} a_{\mathbf{k}, \sigma}+\sum_{\mathbf{k}, \mathbf{q}, \sigma} V(\mathbf{q}) a_{\mathbf{k}, \sigma}^{\dagger} a_{\mathbf{k}-\mathbf{q}, \sigma}
$$

where $a_{\mathbf{k}, \sigma}^{\dagger}, a_{\mathbf{k}, \sigma}$ denote creation and annihilation operators for the electron with wavenumber $\mathbf{k}$ and spin $\sigma, \mu$ is the chemical potential, $V(\mathbf{q})$ is the Fourier transform of the electronimpurity scattering potential, and $\epsilon_{\mathbf{k}}=\mathbf{k}^{2} / 2 m$ is with $m$ the electron mass. In this section we use units such that $\hbar=1$.

We will use the standard Edwards diagram technique.20 Accordingly, we consider retarded (R) and advanced (A) zero-temperature Green's functions,

$$
\mathcal{G}_{\mathbf{k}, \mathbf{p}}^{R, A}(\omega)=\left\langle\mathbf{k}\left|\frac{1}{\omega-H \pm i 0}\right| \mathbf{p}\right\rangle
$$

and their impurity averaged counterparts,

$$
\left\langle\mathcal{G}_{\mathbf{k}, \mathbf{p}}^{R, A}(\omega)\right\rangle=\delta_{\mathbf{k}, \mathbf{p}} \frac{1}{\omega+\epsilon_{F}-\epsilon_{\mathbf{k}}+\Sigma_{\mathbf{k}}^{R, A}(\omega)},
$$

where $\epsilon_{F}=\mu(T=0)$ is the Fermi energy, and $\Sigma_{\mathbf{k}}^{R, A}(\omega)$ is the self energy. $\omega$ measures the energy distance from the Fermi surface. Ultimately we will take the limit $\omega \rightarrow 0$ to obtain the static mobility or conductivity.

We will restrict our considerations to pure s-wave scattering, which is equivalent to the assumption of point-like scatterers. This approximation will substantially simplify all calculations. Its justification and limitations will be discussed in Sec. [II]. For pure s-wave scattering the self-energy reads to linear order in the impurity density,

$$
\Sigma_{\mathbf{k}}^{R, A}(\omega)=\Delta \pm i / 2 \tau+O\left(n^{2}\right)
$$

with $\tau=l m / k_{F}$ the mean-free time. The real part of the self energy, $\Delta$, is to this order independent of the wavenumber $\mathbf{k}$. Even though it is formally infinite for pure s-wave scattering, it therefore strictly renormalizes the chemical potential or the zero of energy, and can be neglected. Although we will want to effectively expand the full Green's function to second order in the impurity density, we will find it convenient to use the self energy as given by Eq. (2.3) and to include higher order contributions to the self energy explicitly at a later stage. We therefore define, for later reference, an auxiliary zero frequency Green's function,

$$
G_{\mathbf{k}}^{R, A}=\frac{1}{\epsilon_{F}-\epsilon_{\mathbf{k}} \pm i / 2 \tau}
$$


We will also need the free electron Green's function,

$$
G_{\mathbf{k}}^{(0) R, A}=\frac{1}{\epsilon_{F}-\epsilon_{\mathbf{k}} \pm i 0}
$$

\section{B. Diagrammatic Expansion of the Conductivity}

It is convenient to calculate the conductivity, $\sigma$, of degenerate electrons at $T=0$, and then to convert to the experimentally relevant finite- $T$ mobility by means of an Einstein relation and a Kubo-Greenwood formula. A general scheme for a diagrammatic calculation of the conductivity within the context of the model defined in Sec. II A above has been given in Ref. 15. The result was a density expansion for the conductivity of the form,

$$
\sigma / \sigma_{B}=1+\sigma_{1} \frac{1}{2 k_{F} l}+\sigma_{2 l o g}\left(\frac{1}{2 k_{F} l}\right)^{2} \ln \left(\frac{1}{2 k_{F} l}\right)+\sigma_{2}\left(\frac{1}{2 k_{F} l}\right)^{2}+O\left(a_{s} / l\right)+o\left(1 /\left(k_{F} l\right)^{2}\right)
$$

with $\sigma_{B}$ the Boltzmann conductivity, $k_{F}$ the Fermi wavenumber, and $a_{s} k_{F}$ considered small. There is no need to repeat the description of the general scheme here. Suffice it to say that the real part of the dynamical conductivity of noninteracting electrons at zero temperature can be expressed in terms of the Green's functions defined in Eq. (2.2) by means of the Kubo-Greenwood formula,

$$
\operatorname{Re} \sigma(\omega)=\frac{e^{2}}{\pi m^{2}} \operatorname{Re} \sum_{\mathbf{k}, \mathbf{p}} v(\mathbf{k})\left\langle\mathcal{G}_{\mathbf{k}, \mathbf{p}}^{R}(\omega) \mathcal{G}_{\mathbf{p}, \mathbf{k}}^{A}(\omega=0)-\mathcal{G}_{\mathbf{k}, \mathbf{p}}^{R}(\omega) \mathcal{G}_{\mathbf{p}, \mathbf{k}}^{R}(\omega=0)\right\rangle v(\mathbf{p})
$$

where $v(\mathbf{k})=\mathbf{k} \cdot \mathbf{q} / q$, with $\mathbf{q}$ an arbitrary fixed vector, is the current vertex. The brackets $\langle\cdots\rangle$ denote the averaging over the random positions of the impurities. We will perform this average by means of standard diagrammatic methods.20 note that this is not the only possible way to calculate the conductivity. One could, for instance, use quantum kinetic theory instead. However, it is known from calculations of the coefficient $\sigma_{1}$ in Eq. (2.5) that the diagrammatic method $\mathbf{5 0}, \mathbf{6}$ leads to much simpler calculations than quantum kinetic theory.21 Technically, our calculation is a systematic extension of the work of Refs. 15 and 16. These authors had already identified all diagrams that contribute to $\sigma_{1}$ and $\sigma_{2 l o g}$, and calculated both coefficients. For our purposes we will now have to calculate the previously considered diagrams to $O\left(n^{2}\right)$ instead of $O\left(n^{2} \ln n\right)$, and to identify and evaluate all diagrams that contribute to $\sigma_{2}$, but neither to $\sigma_{1}$ nor to $\sigma_{2 l o g}$.

Let us start by introducing symbols and abbreviations for the diagrammatic elements of our perturbation theory. The averaged Green's function, Eq. (2.4a), we denote by a directed full line, with an arrow pointing right and left for the retarded and advanced Green's function, respectively. We further denote the difference between the Green's function given by Eq. (2.4a and the free electron Green's function, Eq. 2.4b), by a directed line which carries a triangle. The impurity potential is denoted by a dashed line, and the impurity density by a cross; each cross with a dashed line running through it corresponds to a factor $u=1 / 2 \pi N_{F} \tau$, with $N_{F}=k_{F} m / 2 \pi^{2}$ the free electron density of states per spin at the 
Fermi level. The current vertex $v(\mathbf{k})$ is represented by a triangle. All of these diagrammatic elements can be seen in Fig. 1. We also define the quantities $\epsilon \equiv 2 m \epsilon_{F}$, and $\gamma \equiv m / \tau$.

In terms of these quantities, the simplest diagrammatic contribution to Eq. (2.6) in the limit of zero frequency is the simple bubble shown in Fig. 1(a), and the corresponding diagram with the direction of the lower line reversed. The analytic expression for this contribution to the static conductivity, which we denote by $\sigma_{(1 a)}$, reads,

$$
\sigma_{(1 a)}=\frac{e^{2}}{\pi m^{2}} \operatorname{Re} \sum_{\mathbf{k}}[v(\mathbf{k})]^{2}\left[G_{\mathbf{k}}^{R} G_{\mathbf{k}}^{A}-G_{\mathbf{k}}^{R} G_{\mathbf{k}}^{R}\right] .
$$

Performing the integral is simple, and yields,

$$
\sigma_{(1 a)}=\sigma_{B}\left[1+\frac{3}{2}\left(\frac{\gamma}{2 \epsilon}\right)^{2}+O\left(\left(\frac{\gamma}{\epsilon}\right)^{4}\right)\right] .
$$

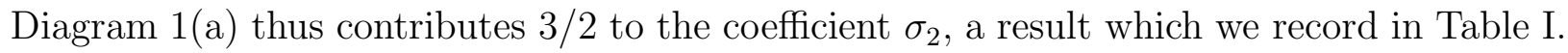
For later reference we also give explicitly the Boltzmann result for the conductivity,

$$
\sigma_{B}=\frac{e^{2} \epsilon^{3 / 2}}{3 \pi^{2} \gamma}=\frac{e^{2} n_{e} \tau}{m}
$$

with the free electron density $n_{e}=\epsilon^{3 / 2} / 3 \pi^{2}$, and $e$ the electron charge. Notice that $\sigma_{B}$ is a function of $\epsilon$, this will be of importance later. We also note that the simple bubble is the only diagram for which we have to consider the $\mathcal{G}^{R} \mathcal{G}^{R}$ contribution to Eq. (2.6). All other diagrammatic contributions to this term turn out to be of higher than second order in the impurity density.

Figure 1 also shows two diagrams that have to be considered due to our choice of the basic Green's function, Eq. 2.4a). If we had calculated the self energy in the basic Green's function to second order in the impurity density these diagrams would not appear. Note the appearance of the 'triangulated' Green's function, which is necessary to avoid double counting. The evaluation of these diagrams is also straightforward, and the results are given in Table $\mathbb{\text { I. }}$.

We now turn to the diagrams that were discussed previously in Refs. 15, 16, and were calculated there to $O\left(n^{2} \ln n\right)$. They are shown again in Fig. 2. We find that all of these diagrams contribute to the analytic term at second order as well. The diagram shown in Fig. 2(a) is of special interest, since for pure s-wave scattering it is found to be ultraviolet divergent. We therefore introduce an ultraviolet cutoff $Q \sim 1 / a_{s}$, and discuss this diagram in some detail. We stress that this divergence is due to an unphysical treatment of the shortrange part of the electron-impurity interaction and often occurs when one uses an s-wave scattering approximation. It should not be confused with the physically more interesting logarithmic singularity in the density expansion of the transport coefficients which is due to long-range collective effects.

The analytic expression corresponding to Fig. 2(a) is,

$$
\begin{array}{r}
\sigma_{(2 a)}=2 u^{2} \sum_{\mathbf{k}, \mathbf{p}, \mathbf{q}}[v(\mathbf{k})]^{2} \operatorname{Re}\left(G_{\mathbf{k}}^{R} G_{\mathbf{k}-\mathbf{p}}^{R} G_{\mathbf{k}-\mathbf{p}-\mathbf{q}}^{R} G_{\mathbf{k}-\mathbf{q}}^{R} G_{\mathbf{k}}^{R} G_{\mathbf{k}}^{A}\right) \\
=\sigma_{B}\left(\frac{\gamma}{2 \epsilon}\right) \frac{1}{2 \pi^{5} \epsilon^{3 / 2}} \operatorname{Re} \int_{0}^{\infty} d q q^{2} J^{++}(q) \\
{\left[(\epsilon-i \gamma)\left[J^{++}(q)-J^{+-}(q)\right]\right.} \\
\left.-(\epsilon+i \gamma) \gamma \frac{d}{d \gamma} J^{++}(q)\right] .
\end{array}
$$


To obtain the second equality we have repeatedly used the identity

$$
G_{\mathrm{k}}^{R} G_{\mathrm{k}}^{A}=\frac{i m}{\gamma}\left(G_{\mathrm{k}}^{R}-G_{\mathrm{k}}^{A}\right)
$$

and defined two functions,

$$
J^{+-}(q)=\int d \mathbf{k} \frac{1}{\epsilon-\mathbf{k}^{2}+i \gamma} \frac{1}{\epsilon-(\mathbf{k}-\mathbf{q})^{2}-i \gamma},
$$

and

$$
J^{++}(q)=\int d \mathbf{k} \frac{1}{\epsilon-\mathbf{k}^{2}+i \gamma} \frac{1}{\epsilon-(\mathbf{k}-\mathbf{q})^{2}+i \gamma}
$$

The same functions had been defined in Refs. 15,116, and all diagrams can be expressed in terms of them. For our present purposes we need a more accurate evaluation of these functions than the one that was given before. It turns out that both integrals can be done exactly in closed form, a task which we relegate to Appendix A. Using the result in Eq. (2.8), a calculation sketched in Appendix B leads to,

$$
\sigma_{(2 a)}=\sigma_{B}\left[-\pi\left(\frac{\gamma}{2 \epsilon}\right)+\left(\frac{\gamma}{2 \epsilon}\right)^{2}(6 \ln (Q / \sqrt{\epsilon})+4-12 \ln 2)+O\left(\left(\frac{\gamma}{2 \epsilon}\right)^{3}\right)\right]
$$

The $\ln Q$-contribution stems from the real part of the self-energy contribution to the upper electron line in diagram 2(a). It thus constitutes just a shift (albeit an infinite one in the case of a pointlike potential) of the chemical potential, like the constant $\Delta$ in Eq. (2.3) which we neglected earlier. We therefore expect the $\ln Q$-term to disappear upon considering the experimentally relevant mobility instead of the conductivity. We will find this expectation to be borne out later, cf. Sec. IIC. The remaining diagrams in Fig. 2 can be calculated along the same lines, and the results are given in Table [4.

The diagrams shown in Figs. 2(d)-(h) allow for generalizations which all contribute to $\sigma_{2}$. These generalizations are obtained by replacing the 'ladder' and 'crossed-ladder' elements in these diagrams by the respective infinite resummations. The resulting diagrams are shown in Fig. 3. Note that the ladder, or 'diffuson', and crossed-ladder, or 'Cooperon', resummations in Fig. 3 start with three rungs each to avoid double counting of the diagrams of Fig. 2. The infinite resummations are again easily expressed in terms of integrals over the functions $J^{++}$ and $J^{+-}$, and the results are listed in Table $\mathbb{1}$. The reason why these diagrams with increasing numbers of impurity lines, and hence increasing numbers of factors $\gamma \sim n$, all contribute to the same order lies in the fact that the diffusion pole contained in the ladder and crossedladder resummations leads, with increasing order, to increasingly singular infrared behavior of the integrand, which is cut off only by $\gamma$. The cancellation of these two effects leads to all of these diagrams being of the same order in $\gamma$ or $n$. By the same argument it follows that these are the only infinite resummations (given our definition of skeleton diagrams by means of Eq. (2.4a)) that contribute to the desired order. In particular, diagrams that contain more than one diffusion pole do not contribute.

We now turn to other skeleton diagram contributions. All relevant diagrams with three impurity lines (as far as they were not included in Fig. 21) are shown in Fig. \&, and all those 
with four impurity lines are shown in Fig. 5. The evaluation of these diagrams offers no particular difficulties, except that some care has to be exercised since the vector nature of the current vertex leads to some nontrivial angular integrations. Of course, all diagrams that contain the same self-energy piece as diagram 2(a) also contain the $\ln Q$-contribution that is characteristic for this diagrammatic element. The results are again given in Table $\mathbb{1}$.

Table [ lists the contributions of all diagrams to the coefficient $\sigma_{2}$ in Eq. (2.5). In some cases we have found it convenient to combine some diagrams before evaluation of the integrals, and this is indicated in the table. The coefficients $\sigma_{1}$ and $\sigma_{2 l o g}$ have been calculated in Ref. 16 with the result,

$$
\sigma_{1}=-4 \pi / 3 \quad, \quad \sigma_{2 l o g}=\left(\pi^{2}-4\right) / 2 .
$$

Table 1 contains three integrals which we could not reduce to tabulated ones, viz.

$$
\begin{gathered}
I_{1}=\int_{0}^{1} \frac{d x}{x}\left[\ln \left(\frac{1-x}{1+x}\right)\right]^{2}=4.207 \ldots \\
I_{2}=\int_{0}^{1} d x x\left[\ln \left(\frac{1-x}{1+x}\right)\right]^{2}=2.772 \ldots \\
I_{3}=\frac{4}{\pi} \int_{0}^{\infty} \frac{d x}{x^{2}}(\arctan x)^{4}\left[1-\frac{1}{x} \arctan x\right]^{-1}=7.716 \ldots .
\end{gathered}
$$

Summing all the contributions listed in Table — we obtain

$$
\sigma_{2}=4 \ln \left(Q / k_{F}\right)+\frac{55}{36} \pi^{2}-14 \ln 2+7-I_{1}+I_{2}-I_{3}=4 \ln \left(Q / k_{F}\right)+3.22 \ldots .
$$

This concludes our calculation of the zero-temperature conductivity. In order to compare with, and make explicit predictions for, experiments it is desirable to convert this result into the corresponding one for the mobility at nonzero temperature. In order to do this, we will need the density of states to second order in the impurity density as well as the conductivity. The density of states is easily obtained from the Green's function via the relation,

$$
N(\epsilon)=-\frac{1}{\pi} \sum_{\mathbf{k}, \mathbf{p}} \operatorname{Im}\left\langle\mathcal{G}_{\mathbf{k}, \mathbf{p}}^{R}(\omega=0)\right\rangle
$$

and to second order it is sufficient to consider the diagrams shown in Fig. 6. The calculation is easy, and we obtain,

$$
N(\epsilon)=N^{(0)}\left[1+\left(\frac{\gamma}{2 \epsilon}\right)^{2}\left(-\frac{1}{2}+2 \ln (Q / 2 \sqrt{\epsilon})-2 \ln 2\right)+O\left(\gamma^{3}\right)\right] .
$$

Here $N^{(0)}=2 N_{F}=m k_{F} / \pi^{2}=m \sqrt{\epsilon} / \pi^{2}$ is the free electron density of states. 


\section{The Electron Mobility}

In a time-of-flight experiment like the one described in Ref. 14 the measured observable is the electron mobility, which is given by $\mu(T)=\sigma(T) / e n(T)$, where $\sigma(T)$ is the temperature dependent conductivity, and $n(T)$ is the electron particle number density. $\sigma(T)$ can be obtained from Eq.(2.5) by means of the Kubo-Greenwood formula, 22 and $n(T)$ from the density of states, Eq. (2.16). We thus have,

$$
\mu(T)=\int_{0}^{\infty} d \epsilon\left(\frac{-\partial f}{\partial \epsilon}\right) \sigma(\epsilon) / e \int_{0}^{\infty} d \epsilon f(\epsilon) N(\epsilon)
$$

with $\sigma(\epsilon)$ and $N(\epsilon)$ from Eqs. (2.5), (2.12), (2.14). and (2.16), respectively, and $f(\epsilon)$ the Fermi function. In writing all of these quantities as functions of $\epsilon=k_{F}^{2}$ one has to keep in mind that the electronic mean free path $l$ is the energy independent parameter which has to be kept fixed. We are interested in the limit of small electron density, where the Fermi function can be replaced by a Boltzmann distribution, $f(\epsilon) \approx \exp (\beta \mu) \exp (-\beta \epsilon)$. Doing the integrals we obtain Eq. (1.3a) for the temperature dependent mobility with $\mu_{1}$ and $\mu_{2 l o g}$ as given by Eqs. (1.3b,1.30), and

$$
\mu_{2}=\frac{1}{16}\left[\frac{\pi^{2}}{36}(55+9 C)-C+8-10 \ln 2-I_{1}+I_{2}-I_{3}\right]-2 \mu_{2 \log } \ln 2=0.236 \ldots,
$$

where $C$ is Euler's constant. As expected (see the discussion after Eq. (2.11)) the mobility is independent of the cutoff $Q$.

\section{DISCUSSION}

Since the mobility is directly measured in a time-of-flight experiment of the type reported in Ref. 14, the density expansion for the mobility as given by Eqs. (1.3), (2.18) can be directly compared with experiment. We split the discussion of the experimental relevance of our result into three separate questions: (1) How accurately does our quantum Lorentz model describe electrons injected into Helium gas? (2) How do our results compare with existing data? (3) What kind of experimental effort would be necessary to unambiguously detect the logarithmic term in the density expansion?

The first question has to be divided into idealizations that are inherent in the model, and effects of additional approximations we have made. Let us start out with the latter. As mentioned in the Introduction, there are three independent length scales in the model, viz. the mean scatterer separation $n^{-1 / 3}$, the mean-free path $l$, and the thermal wavelength $\lambda$. Consequently, one can form four different dimensionless densities, viz. $n \lambda^{3}, n \lambda^{2} a_{s}, n \lambda a_{s}^{2}$, and $n a_{s}^{3}$. The first two do not appear in the Lorentz model.21 Of the other two, the first one is essentially the ratio of the thermal wavelength to the mean-free path, while the second one describes an excluded volume effect that is also present in classical systems. We have kept only the leading (for $\lambda / a_{s}>>1$ ) one of these two parameters, $n \lambda a_{s}^{2}$. That is, we have neglected terms of relative order $a_{s} / \lambda$. At Helium temperature, and with the electronHelium scattering length $\left(a_{s}=0.63 \AA\right)$, the value of this small parameter is $a_{s} / \lambda \approx 10^{-3}$. The prefactor can be estimated from classical Enskog theory, or from the exactly known 
result for the classical Lorentz model,23, and turns out to be of order unity. On the other hand, a typical value for the expansion parameter $\chi$ in Eq. (1.3a) is $\chi \approx 0.1 .4$ In our expansion, excluded volume corrections to the term linear in $\chi$ will therefore roughly be of the same order as terms $\sim \chi^{3}$. We can thus safely neglect excluded volume effects, unless $\chi$ is chosen to be too small, see below. It is also worthwhile to point out that the excluded volume terms have a different temperature dependence than the leading terms that were kept in Eq. (1.3a). In principle it would therefore be possible to separate these contributions experimentally, although this may be hard to do in practice.

Another approximation has been our restriction to pure s-wave scattering. This amounts to neglecting corrections of $O\left(k_{F} / Q\right) \sim O\left(k_{F} a_{s}\right)$ in Eq. (2.5), which translates into corrections of $O\left(a_{s} / \lambda\right)$ in Eq. (1.3a). Non-s-wave scattering effects and excluded volume effects are therefore comparable.

Idealizations that are inherent in the model include the static nature of the scatterers, the assumption that the scatterers are uncorrelated, and the single-electron approximation. The effects of static correlations between the scatterers can be easily estimated from, e.g., Baym's formula for the electron scattering rate, which can be obtained as a variational solution of the Boltzmann equation.24 For the case of static impurities, Baym's formula contains a static impurity structure factor $S(q)$ under the momentum integral that determines the scattering rate. For uncorrelated scatterers, $S(q) \equiv 1$. Deviations of $S(q)$ from unity, and hence static correlations between the scatterers, are again due to excluded volume effects which we have estimated above. The dynamics of the Helium gas lead, at temperatures around $4 K$, to corrections of the same order, which can be seen as follows. A typical value for the scattering time is $\tau \approx 10^{-12} \mathrm{~s}$. During this time the thermal velocity of the Helium atoms leads to a displacement $d$ on the order of $1 \AA$. This is comparable with the scattering length, and the effect on the electron mobility is thus on the order of $d / \lambda \sim a_{s} / \lambda$, which is again the magnitude of the excluded volume effects. Finally, one has to estimate the effects of the Coulomb interaction, since any real experiment has to deal with more than one electron. Let us use the parameters of Schwarz's experiment 4 for this purpose. A typical value for the electron current density was $j \approx 10^{-12} \mathrm{Ccm}^{-2} \mathrm{~s}^{-1}$, and for the drift velocity $v \approx 10^{4} \mathrm{~cm} / \mathrm{s}$. This corresponds to an electron density $n_{e} \approx 10^{3} \mathrm{~cm}^{-3}$. The Coulomb energy $E_{c} \approx e^{2} / n_{e}^{-1 / 3}$ is then less than one percent of the kinetic energy, $k_{B} T$. If desirable, Coulomb effects can be made even smaller by decreasing the electron density.

We conclude from the preceding discussion that both the model and our additional approximations are adequate for a description of electrons in Helium gas in a parameter range of interest to us, namely at temperatures and densities such that our expansion, Eq. (1.3a), is meaningful. Let us now discuss the relation of our result to existing 14 and possible future experiments. The main remaining uncertainties in this relation arise from the terms of $o\left(\chi^{2}\right)$ and $O\left(\chi a_{s} / \lambda\right)$ in Eq. (1.3a). The former are undoubtedly nonanalytic, but neither their functional form nor their magnitude are known.25 In order to estimate their importance we therefore have to rely on some assumptions. First, it is plausible to neglect the nonanalyticity, and to assume that the coefficients in the $\chi$-expansion are all of roughly the same magnitude. In the Kubo-Greenwood integration, Eq. (2.17), the $\chi^{3}$-term picks up an extra factor of $\sqrt{\pi}$, and so we estimate $o\left(\chi^{2}\right) \approx \mu_{3} \chi^{3}$ with $-2 \sqrt{\pi} \mu_{2} \lesssim \mu_{3} \lesssim 2 \sqrt{\pi} \mu_{2}$. Here we have neglected the, presumably weak, $\chi$-dependence of $\mu_{3}$, and have allowed for a safety margin in the form of an extra factor of 2 . Obviously, the relative effects of $\mu_{3}$, 
and the other unknown terms of higher order, become smaller with decreasing values of $\chi$. On the other hand, the excluded volume effects are of $O\left(\chi a_{s} / \lambda\right)$, and become important if $\chi$ becomes very small. However, as long as they are small compared to the second order terms which we keep in Eq. (1.3a), i.e. as long as $\chi^{2}>\chi a_{s} / \lambda$, or $\chi>a_{s} / \lambda$, they can be neglected. This means there is a window of $\chi$-values for which the excluded volume terms are negligible, but the higher order in $\chi$-terms are not yet important.

We now define, as a convenient quantity directly comparable with experiment,

$$
f(\chi) \equiv\left[\mu(T) / \mu_{B}-1-\mu_{1} \chi\right] / \chi^{2} .
$$

Our theoretical prediction for this quantity is,

$$
f(\chi)=\mu_{2 \log } \ln \chi+\mu_{2} \pm \mu_{2} 2 \sqrt{\pi} \chi
$$

with $\mu_{2}$ from Eq. (2.18), and $\mu_{2 l o g}$ from Eq. (1.30). Let us consider the experimental results obtained by Schwarz 14 at Helium temperature. At $T=4.2 \mathrm{~K}$, a He gas density $n=10^{21} \mathrm{~cm}^{-3}$ corresponds to $\chi=1$, and data were obtained for $\chi$ as low as 0.08. In Fig. 7 we show the theoretical prediction, Eqs. (3.1), for $0<\chi<0.7$ together with Schwarz's data. The error bars shown assume a total error of $3 \%$ in $\mu / \mu_{B}$ and $4 \%$ in $\chi$. To illustrate the effect of the logarithmic term the figure also shows what the theoretical prediction would be if $\sigma_{2 l o g}$ in Eq. (2.5) was zero.

We are now in a position to draw the following conclusions. (1) The existing data are certainly consistent with the existence of the logarithmic term, but are not accurate enough to be conclusive. (2) A repetition of this experiment in the region $0.1<\chi<0.2$ with an accuracy improved by at least a factor of 10 would be sufficient for a convincing test of the logarithmic term's existence. This range of $\chi$-values is particularly suitable because excluded volume effects are negligible. At larger $\chi$-values the uncertainty due to the $\chi^{3}$-terms makes the theoretical prediction meaningless, and at lower $\chi$-values errors in determining $\chi$ translate into very large errors in $f(\chi)$. Also, the excluded volume effects become noticable at smaller $\chi$. (3) The type of experiment discussed, i.e. a time-of-flight measurement for electrons injected into Helium gas, probably constitutes the most favorable opportunity for an experimental check of the existence or otherwise of the logarithmic term in the density expansion for transport coefficients. We hope that the foregoing discussion will stimulate a new precision experiment on this system.

\section{ACKNOWLEDGMENTS}

We gratefully acknowledge helpful discussions with P.W.Adams and J.V.Sengers. One of us (KIW) is grateful for the hospitality extended to him during a stay at the University of Oregon. This work was supported by the NSF under grant numbers DMR-92-17496 and DMR-92-09879.

\section{APPENDIX A: EVALUATION OF THE INTEGRALS $J^{++}(\mathbf{q})$ AND $J^{+-}(\mathbf{q})$}

We have seen in Section II that the diagrams can be conveniently expressed in terms of the two integrals $J^{++}(q)$ and $J^{+-}(q)$ given by Eqs. (2.10). In Refs. 15, 16 approximate 
representations for these integrals were given, which are not sufficient for the purposes of the present paper. We therefore evaluate $J^{++}(q)$ and $J^{+-}(q)$ exactly. The method we are using actually allows us to calculate two slightly more complicated functions, viz.

$$
J^{++}(q, \omega)=\int d \mathbf{k} \frac{1}{\epsilon-\mathbf{k}^{2}+i \gamma} \frac{1}{\epsilon+\omega-(\mathbf{k}-\mathbf{q})^{2}+i \gamma},
$$

and

$$
J^{+-}(q, \omega)=\int d \mathbf{k} \frac{1}{\epsilon-\mathbf{k}^{2}+i \gamma} \frac{1}{\epsilon+\omega-(\mathbf{k}-\mathbf{q})^{2}-i \gamma} .
$$

$J^{++}(q)$ and $J^{+-}(q)$ are obtained as the values of these integrals at $\omega=0$.

To proceed we introduce spherical coordinates $(k, \theta, \phi)$. The integral over the azimuthal angle $\phi$ is trivial and gives a factor of $2 \pi$. The integration over the polar angle $\theta \in[0, \pi]$ can be written as an integral over $x=\cos \theta$ extending from -1 to +1 . Using the symmetry of the integrand then allows us to write

$$
J^{+\nu}(q, \omega)=2 \pi \int_{0}^{1} d x \int_{0}^{\infty} d k \frac{k^{2}}{\alpha-k^{2}}\left[\frac{1}{\beta_{\nu}-k^{2}-2 k q x}+\frac{1}{\beta_{\nu}-k^{2}+2 k q x}\right]
$$

where $\alpha=\epsilon+i \gamma, \beta_{\nu}=\epsilon+\omega+i \nu \gamma-q^{2}$ and $\nu= \pm 1$. Since the integrand is an even function of $k$, one can now extend the $k$-integration to the interval $[-\infty, \infty]$, and evaluate the integral by means of the residue theorem. The remaining integrals over $x$ are elementary, and one finds,

$$
\begin{array}{r}
J^{+\nu}(q, \omega)=\nu \frac{i \pi^{2}}{2 q}\left[\ln \left(\frac{\sqrt{q^{2}+\beta_{\nu}}+q}{\sqrt{q^{2}+\beta_{\nu}}-q}\right)\right. \\
\left.-\ln \left(\frac{2 \nu \sqrt{\alpha} \beta_{\nu}+q\left(\alpha-\beta_{\nu}\right)+\left(\alpha+\beta_{\nu}\right) \sqrt{q^{2}+\beta_{\nu}}}{2 \nu \sqrt{\alpha} \beta_{\nu}-q\left(\alpha-\beta_{\nu}\right)+\left(\alpha+\beta_{\nu}\right) \sqrt{q^{2}+\beta_{\nu}}}\right)\right] .
\end{array}
$$

After some simplifications we obtain,

$$
J^{++}(q, \omega)=-\frac{i \pi^{2}}{q} \ln \left(\frac{\sqrt{\epsilon+\omega+i \gamma}+\sqrt{\epsilon+i \gamma}-q}{\sqrt{\epsilon+\omega+i \gamma}+\sqrt{\epsilon+i \gamma}+q}\right),
$$

and,

$$
J^{+-}(q, \omega)=\frac{i \pi^{2}}{q} \ln \left(\frac{\sqrt{\epsilon+\omega-i \gamma}-\sqrt{\epsilon+i \gamma}-q}{\sqrt{\epsilon+\omega-i \gamma}-\sqrt{\epsilon+i \gamma}+q}\right) \quad .
$$

In the limit $\omega \rightarrow 0, J^{+-}$can be further simplified, and we finally obtain,

$$
J^{++}(q)=\frac{i \pi^{2}}{q} \ln \left(\frac{2 \sqrt{\epsilon+i \gamma}+q}{2 \sqrt{\epsilon+i \gamma}-q}\right)
$$

and

$$
J^{+-}(q)=\frac{2 \pi^{2}}{q} \arctan \left(\frac{q}{2 \operatorname{Im} \sqrt{\epsilon+i \gamma}}\right)
$$


For small $\gamma$ these expressions agree with those obtained previously 16

For explicit perturbative calculations the following formal small- $\gamma$ expansions are useful,

$$
\begin{gathered}
J^{++}(q)=\frac{\pi^{3}}{q} \Theta(q-2 \sqrt{\epsilon})-i \frac{\pi^{2}}{q} \ln \left|\frac{q-2 \sqrt{\epsilon}}{q+2 \sqrt{\epsilon}}\right|+\frac{\gamma}{\epsilon}\left(\frac{2 \pi^{2} \sqrt{\epsilon}}{4 \epsilon-q^{2}}-i \pi^{3} \frac{\sqrt{\epsilon}}{q} \delta(q-2 \sqrt{\epsilon})\right)+O\left(\gamma^{2}\right) \\
J^{+-}(q)=\frac{\pi^{3}}{q}-\frac{\gamma}{\sqrt{\epsilon}} \frac{2 \pi^{2}}{q^{2}}+O\left(\gamma^{2}\right) .
\end{gathered}
$$

\section{APPENDIX B: EVALUATION OF DIAGRAM 2(a)}

To calculate the contribution of diagram 2(a) to the zero-temperature conductivity we use the expressions for $J^{++}(q)$ and $J^{+-}(q)$ from Appendix @ in Eq. (2.8), which we rewrite as,

$$
\begin{array}{r}
\sigma_{(2 a)}=\sigma_{B}\left(\frac{\gamma}{2 \epsilon}\right) \frac{1}{2 \pi^{5} \epsilon^{3 / 2}}\left[\operatorname{Re}(-i \gamma) \int_{0}^{Q} d q q^{2}\left(J^{++}(q)\right)^{2}\right. \\
+\operatorname{Re}(i \gamma) \int_{0}^{Q} d q q^{2} J^{++}(q) J^{+-}(q)-\epsilon \operatorname{Re} \int_{0}^{Q} d q q^{2} J^{++}(q) J^{+-}(q) \\
\left.-\epsilon \gamma \operatorname{Re} \int_{0}^{Q} d q q^{2} J^{++}(q) \frac{d}{d \gamma} J^{++}(q)+\epsilon \operatorname{Re} \int_{0}^{Q} d q q^{2}\left(J^{++}(q)\right)^{2}\right]+O\left(\gamma^{2}\right) .
\end{array}
$$

Because there is a factor $\gamma / 2 \epsilon$ multiplying the integrals it is sufficient to evaluate the latter to linear order in $\gamma$. Of the five integrals the first two can be straightforwardly evaluated by using the expansion given in Eq. (A6). The same is true for the third integral, although the expansion procedure leads to an ill-defined integral $\int_{0}^{2} d x /(x-1)$, which has to be interpreted in a principal values sense (so it is zero). Alternatively, one can use the exact expressions for $J^{++}$and $J^{+-}$from Eqs. (A5), write the arctan as an auxiliary integral, $\arctan x=x \int_{0}^{1} d y /\left(1+y^{2} x^{2}\right)$, and use complex analysis. The result is the same, viz.

$$
\operatorname{Re} \int_{0}^{Q} d q q^{2} J^{++}(q) J^{+-}(q)=\pi^{6}(Q-2 \operatorname{Re} \sqrt{\epsilon+i \gamma})-\left(\gamma 4 \pi^{5} / \sqrt{\epsilon}\right) \ln (Q / 2 \sqrt{\epsilon})+O\left(\gamma^{2}\right)
$$

where we have dropped terms that vanish for $Q \rightarrow \infty$. The fourth term requires the evaluation of an integral,

$$
J \equiv \lim _{\delta \rightarrow 0} \operatorname{Im} \int_{-Q}^{Q} d q q \ln \left(\frac{2 \sqrt{\epsilon+i \gamma}+q}{2 \sqrt{\epsilon+i \gamma}-q}\right) \frac{1}{q+2 \sqrt{\epsilon+i \gamma}+\delta} \frac{1}{q-2 \sqrt{\epsilon+i \gamma}-\delta},
$$

where we have made use of the symmetry of the integrand, and have shifted the poles off the branch cuts of the logarithm. Standard complex analysis techniques yield, in the limit of large $Q$,

$$
J=-2 \pi[\ln (Q / 2 \sqrt{\epsilon})-\ln 2]+O\left(\gamma^{2}\right)
$$

Finally, the fifth integral can be related to the fourth one by Taylor expanding in $\gamma$. Adding all contributions one obtains entry 2(a) in Table 1 . 


\section{REFERENCES}

${ }^{1}$ For a recent review see, e.g., J. R. Dorfman, T. R. Kirkpatrick, and J. V. Sengers, 'Generic long-range correlations in molecular fluids', to appear in Annual Reviews of Physical Chemistry.

${ }^{2}$ R. Peierls, Surprises in Theoretical Physics, Princeton University (Princeton 1979), ch. 5.1.

${ }^{3}$ N. N. Bogoliubov, in Studies in Statistical Mechanics, vol.1, edited by G. E. Uhlenbeck and J. de Boer, North Holland (Amsterdam 1961), p.1.

${ }^{4}$ See, e.g., G. Wannier, Statistical Physics, Dover (New York 1987), ch. 12.

${ }^{5}$ See, e.g., J. P. Boon and S. Yip, Molecular Hydrodynamics, Dover (New York 1991).

${ }^{6}$ J. R. Dorfman and E. G. D. Cohen, Phys. Lett. 16, 124 (1965); J. Math. Phys. 8, 282 (1967); J. Weinstock, Phys. Rev. 140A, 460 (1965). For further references see, e.g., J. R. Dorfman and H. van Beijeren in Statistical Mechanics, part B, edited by B. J. Berne, Plenum (New York 1977), p.65.

${ }^{7}$ J. V. Sengers, Phys. Rev. Lett. 15,515 (1965); K. Kawasaki and I. Oppenheim, Phys. Rev. 139A, 1763 (1965); see also E. G. D. Cohen in Statistical Mechanics at the Turn of the Century, edited by E. G. D. Cohen, Marcel Dekker (New York 1971), p.33, and Ref. 1, and references therein.

${ }^{8}$ C. Bruin, Physica 72, 261 (1974).

${ }^{9}$ J. S. Langer and T. Neal, Phys. Rev. Lett. 16, 984 (1966); J. Weinstock, Phys. Rev. Lett. 17, 130 (1966); see also P. Resibois and M. G. Velarde, Physica 51, 541 (1971).

${ }^{10}$ A list of experiments on classical fluids that tried to detect the logarithmic term can be found in B. M. Law and J. V. Sengers, J. Stat. Phys. 57, 531 (1989).

${ }^{11}$ See, e.g., P. A. Lee and T. V. Ramakrishnan, Rev. Mod. Phys. 57, 287 (1985).

${ }^{12}$ See, e.g., D. Belitz and T. R. Kirkpatrick, Rev. Mod. Phys. 66, 261 (1994).

${ }^{13}$ For a discussion of Lorentz models, see, E. H. Hauge, in Transport Phenomena, Lecture Notes in Physics No. 31, edited by G. Kirczenow and J. Marro, Springer (New York 1974), p.337.

${ }^{14}$ K. Schwarz, Phys. Rev. B 21, 5125 (1980), and references therein.

${ }^{15}$ T. R. Kirkpatrick and J. R. Dorfman, Phys. Rev. A 28, 1022 (1983). See also Ref. 16 which corrected an algebraic error in the results of this paper.

${ }^{16}$ T. R. Kirkpatrick and D. Belitz, Phys. Rev. B 34, 2168 (1986).

${ }^{17}$ P. W. Adams, D. A. Browne, and M. A. Paalanen, Phys. Rev. B 45, 8837 (1992).

${ }^{18}$ See, e.g., the discussion in Ref. 12, Sec. II.A.1.d.

${ }^{19}$ K. I. Wysokinski, W. Park, D. Belitz, and T. R. Kirkpatrick, Phys. Rev. Lett. xx, xxxx (1994).

${ }^{20}$ See, e.g., A. A. Abrikosov, L. P. Gorkov, and I. E. Dzyaloshinskii, Methods of Quantum Field Theory in Statistical Physics Prentice Hall (Englewood Cliffs, 1969), ch. 39; or G. D. Mahan Many-Particle Physics (Plenum Press, New York and London, 1981), ch. 7.

${ }^{21}$ T. R. Kirkpatrick and J. R. Dorfman, J. Stat. Phys. 30, 67 (1983).

${ }^{22}$ D. A. Greenwood, Proc. Phys. Soc. 71, 585 (1958).

${ }^{23}$ J. M. J. van Leeuwen and A. Weijland, Physica 36, 457 (1967).

${ }^{24}$ G. Baym, Phys. Rev. 135, A1691 (1964).

${ }^{25}$ Our calculations suggest that the leading nonanalyticity at third order in $\chi$ is of the form $\chi^{3}(\ln \chi)^{2}$. 


\section{FIGURES}

FIG. 1. The simple bubble (a), and two diagrams containing the 'triangulated' Green's function (b), (c). Together with diagrams (b) and (c), their complex conjugates (c.c.) also contribute.

FIG. 2. The diagrams that were considered previously in Refs. 15,16.

FIG. 3. Infinite resummations that are derivatives of diagrams 2(d)-2(h).

FIG. 4. Skeleton diagrams with three impurity lines

FIG. 5. Skeleton diagrams with four impurity lines

FIG. 6. The Green's function to second order in the impurity density

FIG. 7. The reduced mobility $f$, as defined in Eq. (3.19), vs. the density parameter $\chi=\lambda / \pi l$. The theoretical prediction is for $f$ to lie between the two solid lines. The experimental data are from Fig. 9 of Ref. 14 with error bars estimated as described in the text. The broken lines show

what the theoretical prediction would be in the absence of the logarithmic term in the density expansion. 


\section{TABLES}

TABLE I. Values of the diagrams as shown in Figs. 1-15. Only the contribution to the coefficient $\sigma_{2}$ in Eq. (2.5) is given, for contributions to lower orders see Refs. 15,16. See the text for further explanation

\begin{tabular}{cccc}
\hline \hline diagram & $\sigma_{2}$ & diagram & $\sigma_{2}$ \\
\hline 1 (a) & $3 / 2$ & $4(\mathrm{f})$ & $-I_{1}+2 I_{2}$ \\
1 (b) & $-7 / 2$ & $4(\mathrm{~g})$ & $4 \ln 2-2$ \\
1 (c) & 1 & $4(\mathrm{~h})$ & $2-4 \ln 2$ \\
2 (a) & $6 \ln Q / \sqrt{\epsilon}+4-12 \ln 2$ & $4(\mathrm{i})$ & $I_{1} / 2-I_{2}$ \\
2 (b) & 8 & $5(\mathrm{a})+(\mathrm{b})$ & 0 \\
2 (c) & $-2-4 \ln 2$ & $5(\mathrm{c})+(\mathrm{d})$ & 0 \\
2 (d) & $\pi^{2} / 2+3 I_{1} / 2$ & $5(\mathrm{e})$ & $\pi^{2} / 9$ \\
$2(\mathrm{e})+(\mathrm{f})+(\mathrm{g})+(\mathrm{h})$ & $-1-\pi^{2}-2 \ln 2$ & $5(\mathrm{f})$ & 0 \\
$3(\mathrm{a})+(\mathrm{b})+(\mathrm{c})$ & $\pi^{2} / 4-2 \ln 2$ & $5(\mathrm{~g})$ & $2 \pi^{2} / 3$ \\
3 (d) & $-I_{3}$ & $5(\mathrm{~h})+(\mathrm{i})$ & $\pi^{2}$ \\
4 (a) & $-I_{1} / 2$ & $5(\mathrm{l})+(\mathrm{m})$ & 0 \\
4 (b) & $-I_{1} / 2$ & $5(\mathrm{n})+(\mathrm{o})$ & 0 \\
$4(\mathrm{c})$ & $-I_{1}$ & $5(\mathrm{p})$ & 0 \\
$4(\mathrm{~d})$ & $-2 \ln (Q / \sqrt{\epsilon})+4 \ln 2$ & & 0 \\
$4(\mathrm{e})$ & $2 \ln 2-1$ & & \\
\hline \hline
\end{tabular}




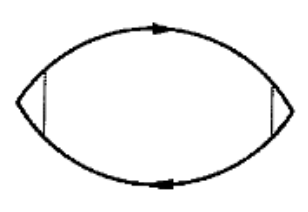

(a)

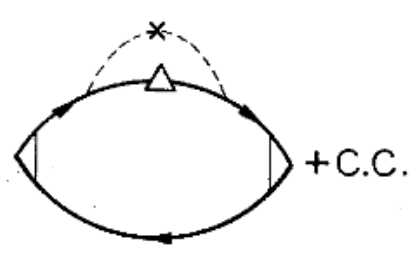

(b)

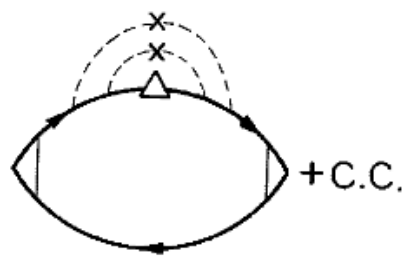

(c)

$\overrightarrow{\vec{k}} \triangle \longrightarrow=G_{\vec{k}}^{R}-G_{\vec{k}}^{(0) R}$

Fig. 1 


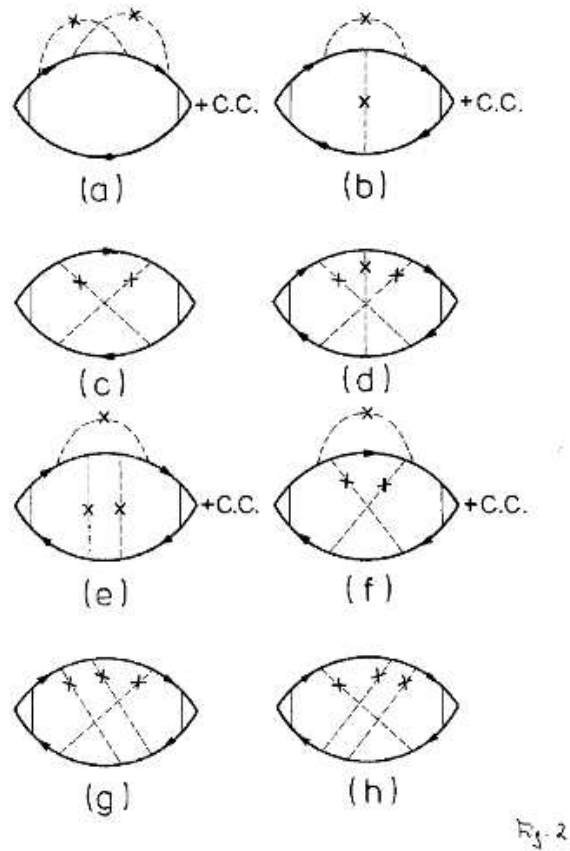




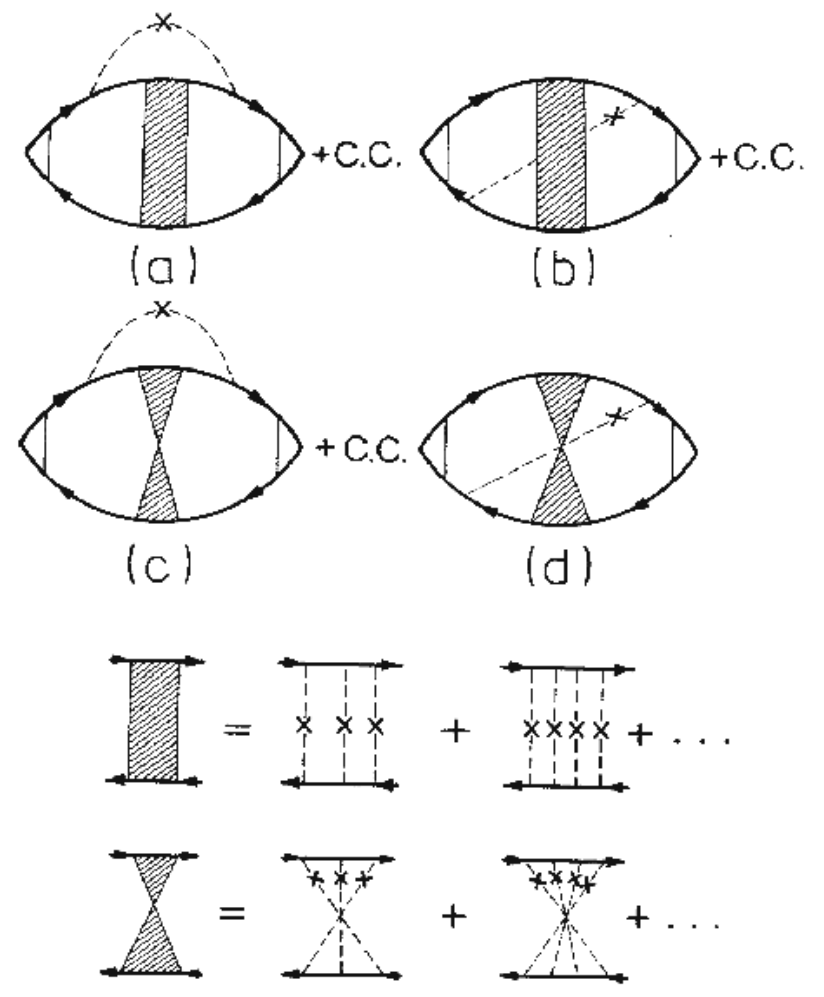

Fig. 3 


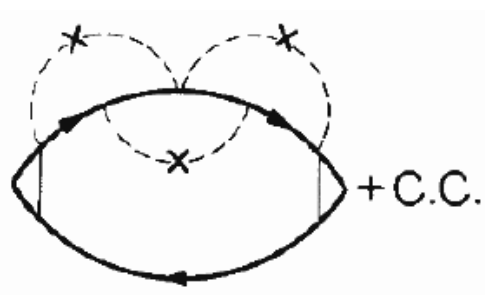

(a)

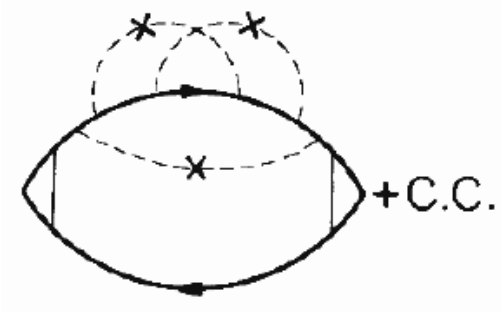

(d)

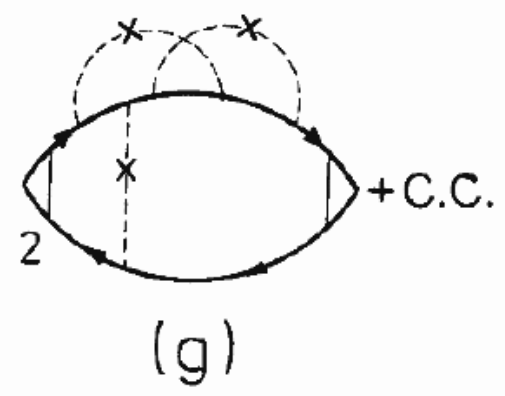

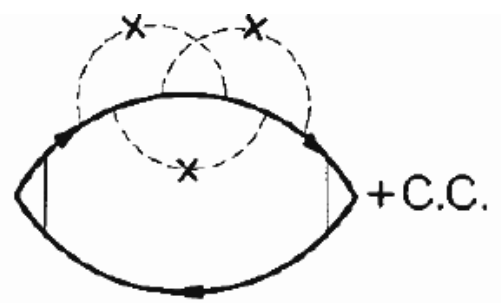

(b)

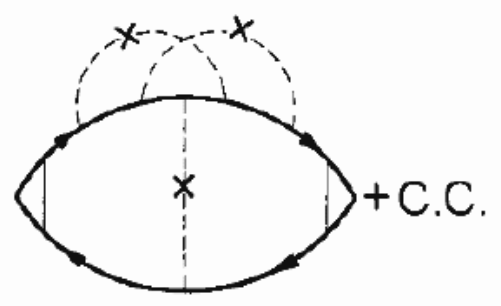

(e)

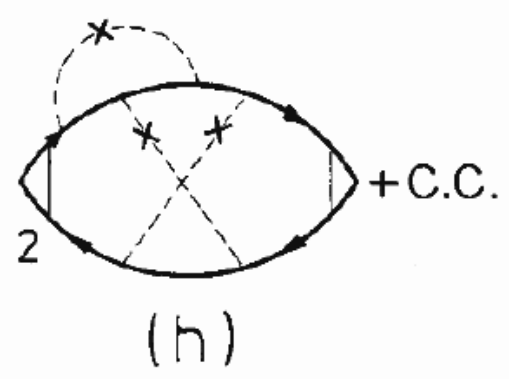

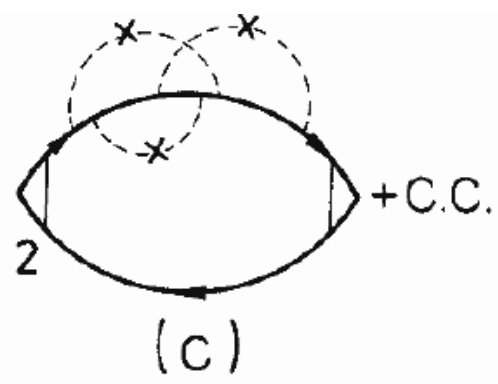
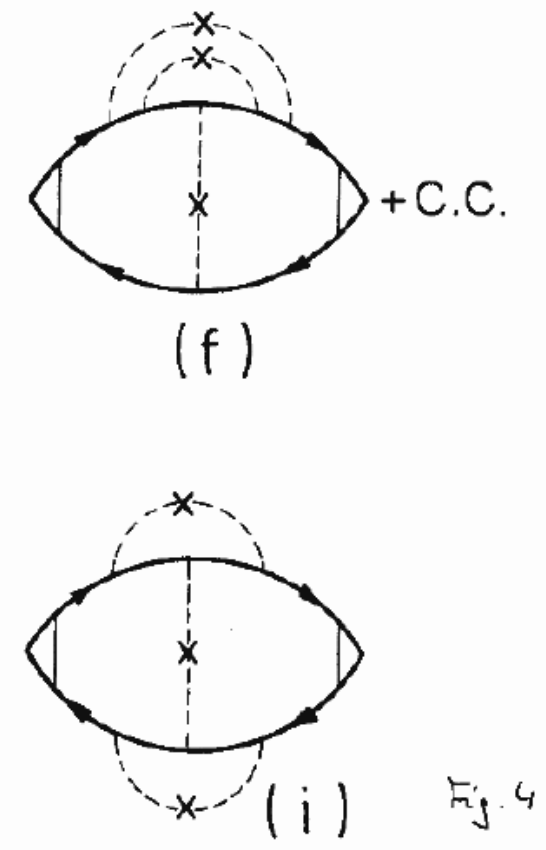


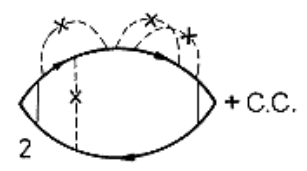

(a)

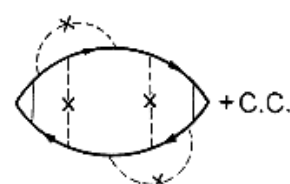

(d)

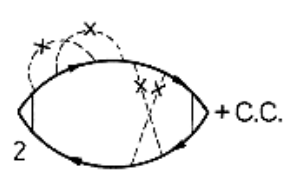

(g)

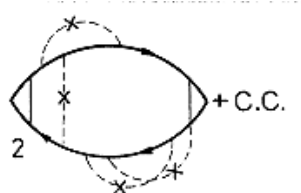

(b)

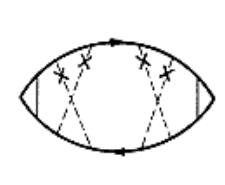

(e)

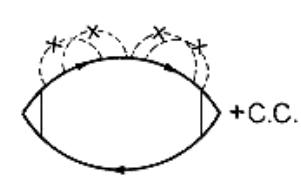

(h)

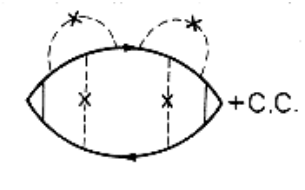

(c)

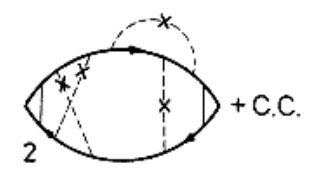

(f)

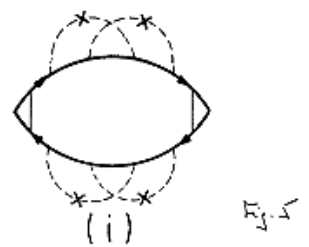



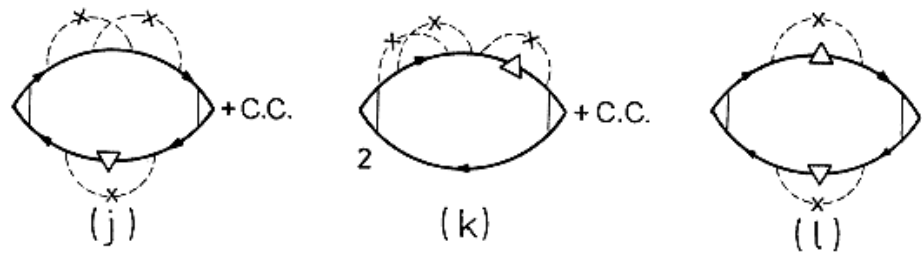

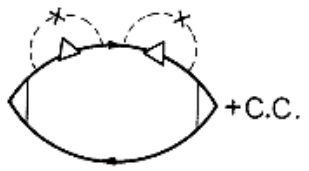

(m)

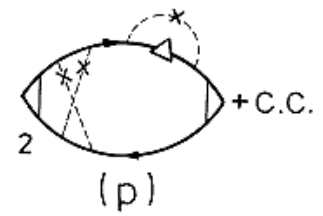

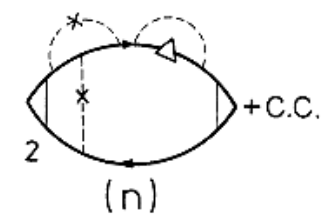

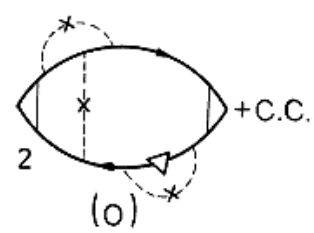

Fig.5 ctd. 


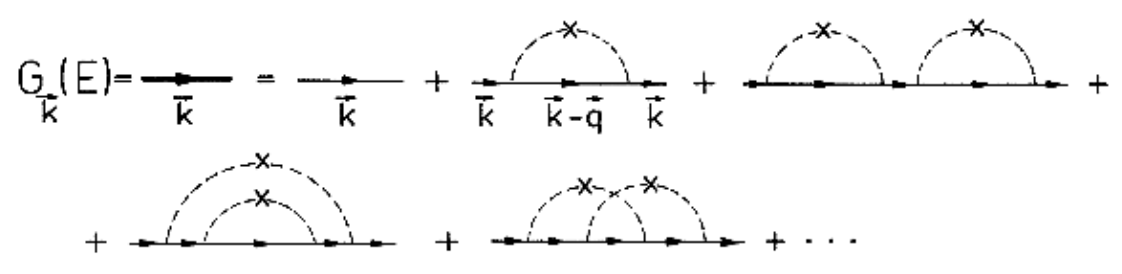

$F_{i j} .6$ 


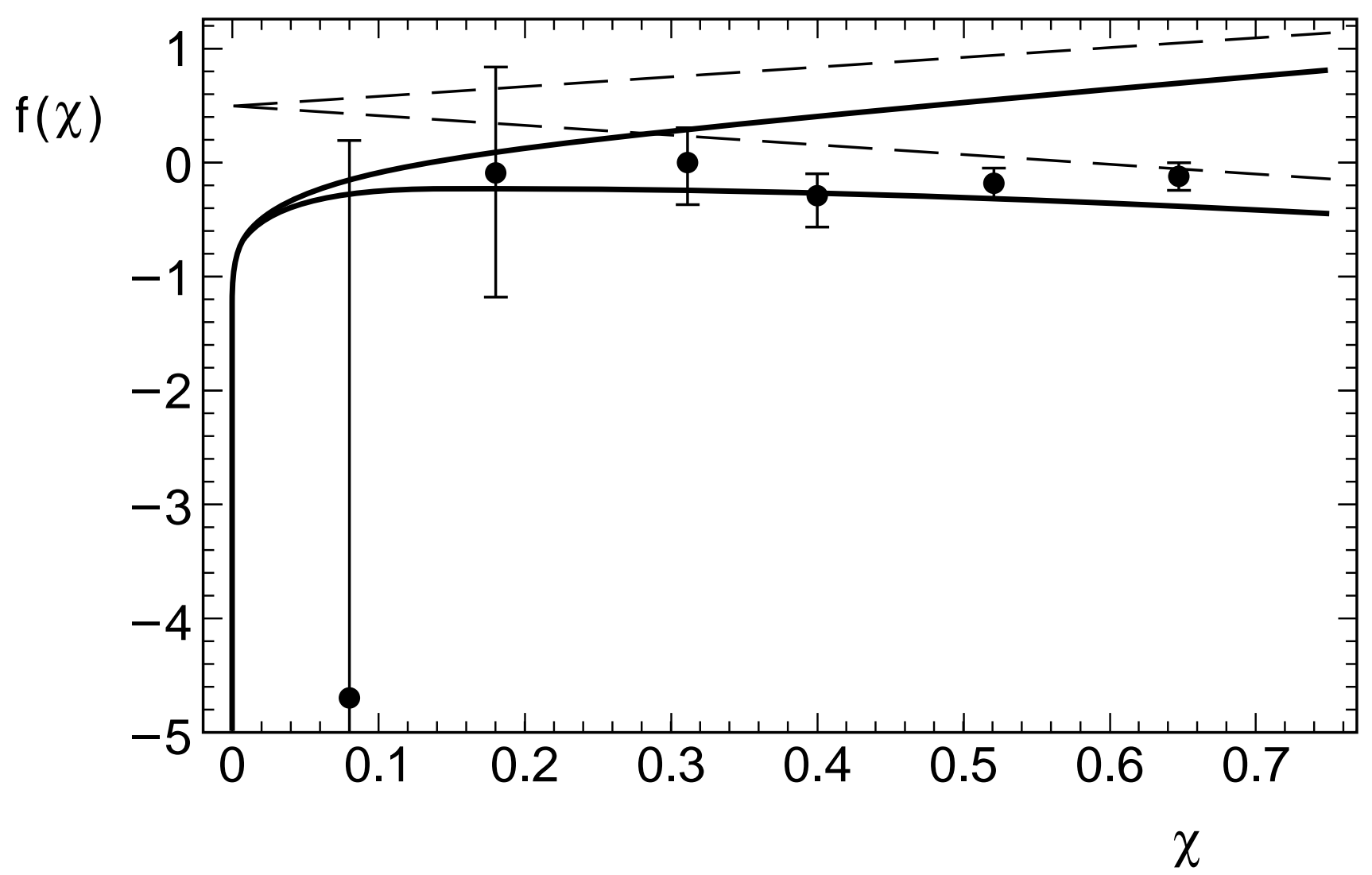

